\title{
Automated Classification and Analysis of Axons and Dendrites of Hippocampal Neurons in Cell Culture
}

\author{
M.-A. Abdul-Karim,* N. M. Dowell-Mesfin, ${ }^{* *}$ W. Shain, ${ }^{* *}$ J. N. Turner,** and B. Roysam* \\ * Rensselaer Polytechnic Institute, Troy, NY 12180 \\ ** The Wadsworth Center, New York State Department of Health, Albany, NY 12201
}

An increasing body of evidence suggests significant differences in the growth and differentiation of axons and dendrites in neurons. Automated methods to classify process type would greatly facilitate studying these differences. We have developed adaptive image analysis and registration tools, and type-specific markers for dendrites and axons to segment exclusively each process type for detailed quantitative morphometric analysis.

Hippocampal neurons were grown in vitro on poly-lysine coated silicon surfaces, fixed and immunolabeled for MAP-2 and B-III Tubulin proteins. MAP2+ labeling was confined to cell somas and more proximal dendrites MAP2+ (Fig. 1a), while and Tubulin+ labeling labeled the somas and all processes (Fig. 1b). Images were acquired using epifluorescence microscopy, utilizing separate filter sets to acquire the MAP2+ and Tubulin+ data sets.

An automated tracing algorithm was utilized to segment the processes, producing centerlines and statistics (lengths, directionality, widths, and counts) [1]. The algorithm is fast, taking just 2 seconds to trace the entire $1280 \times 1024$ image field on a Pentium III, $1 \mathrm{GHz}$ personal computer. MAP2+ and Tubulin+ images were traced separately. Using the resulting two sets of centerlines as the set of correspondences, the traces were registered [2] to counter spatial distortion due to usage of dual filters. From here, MAP2+ and Tubulin+ processes or portions of processes were identified (Fig. 1c). These neurons were cultured over 14 days on nanofabricated surfaces with a rectangular matrix of pillars ranging in widths from 0.5-2.0 $\mu \mathrm{m}$, with spacing between 1.5-4.5 $\mu \mathrm{m}$. The resulting images, and automated tracing results superimposed (in green) are shown in Figure 2. The traces reveal significant differences and trends in the angular growth patterns of the neurons, which were quantified using angular histograms (Fig. 2c).

In the near term, inclusion of morphological and additional biological information such as presynaptic and postsynaptic markers will be tested to improve our abilities to distinguish between dendrites and axons. Nevertheless, due to the speed, accuracy, and clutter-tolerance of the tracing algorithm utilized for neuron process-type classification, this framework is suitable for large-scale morphological studies targeted to produce statistically significant results.

References

[1] K. A. Al-Kofahi et al., IEEE Trans. Inform. Technol. Biomed. 6(2) (2002) 171.

[2] P. J. Besl and N. D. McKay, IEEE Trans. Pattern Anal. Mach. Intell., 14(2) (1992) 239.

[3] A. Caceres et al., Brain Res. 315(2) (1984) 314.

[4] This work is supported by the NSF Center for Subsurface Sensing \& Imaging Systems (EEC9986821), MARA of Malaysia, the NSF Nanobiotechnology Center (ECS-9876771), Microbrightfield Inc., and NIBIB (EB-00359). 


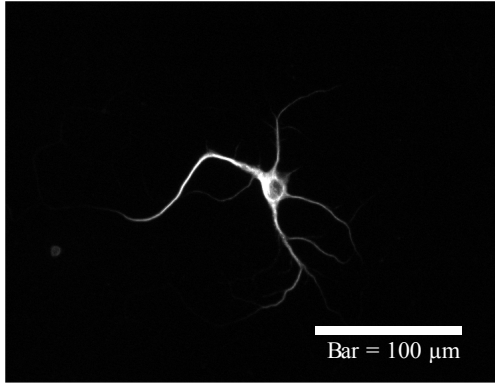

(A)

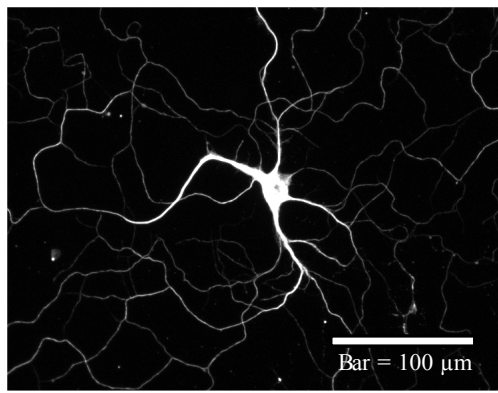

(B)

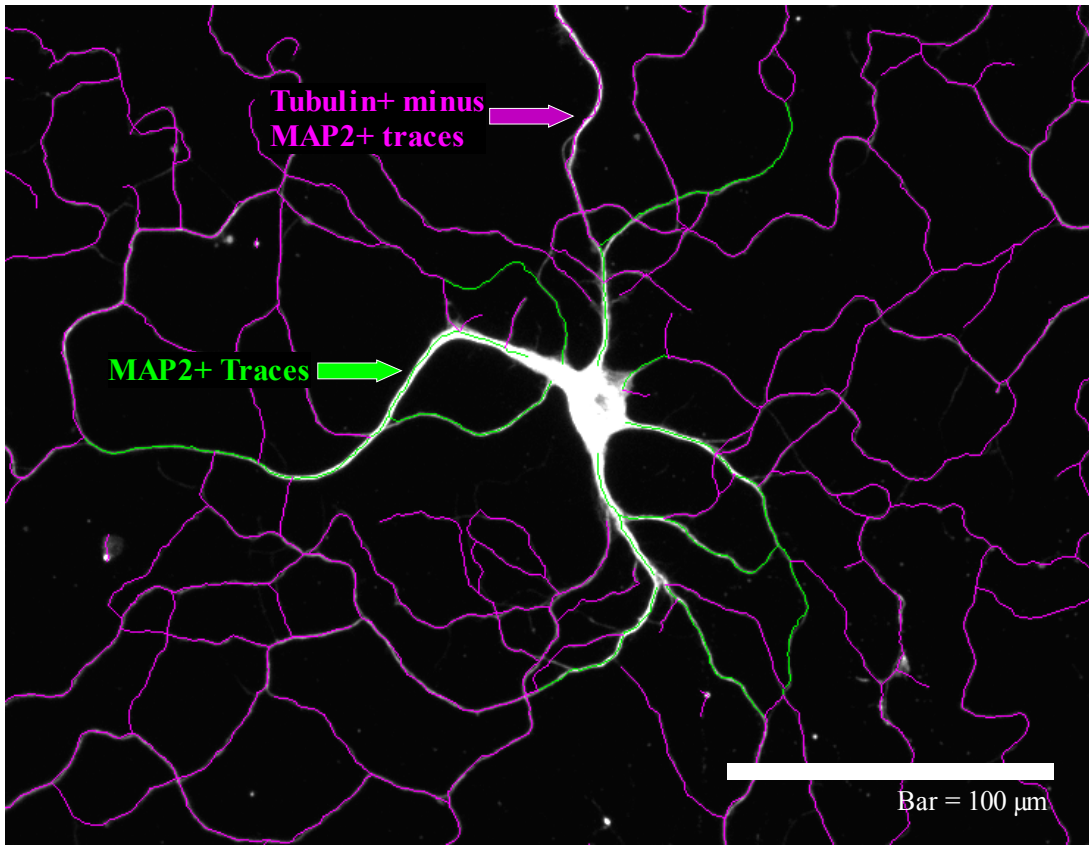

(C)

FIG 1: (A) Image showing the distribution of MAP-2 protein in neurites, apparently more prominent in dendrites than axons. (B) An image showing the distribution of $\beta$-III Tubulin in neurites. Note that it contains both neurites with dense MAP-2 protein and obvious axons. (C) The resulting registered traces of neurites traced from images shown in (A) and (B). Clearly that information from MAP2+ and Tubulin+ neurites are insufficient to distinguish dendrites and axons at this point. Ongoing efforts to include more morphological and biological information such as presynaptic and postsynaptic sites will make the distinction much clearer.

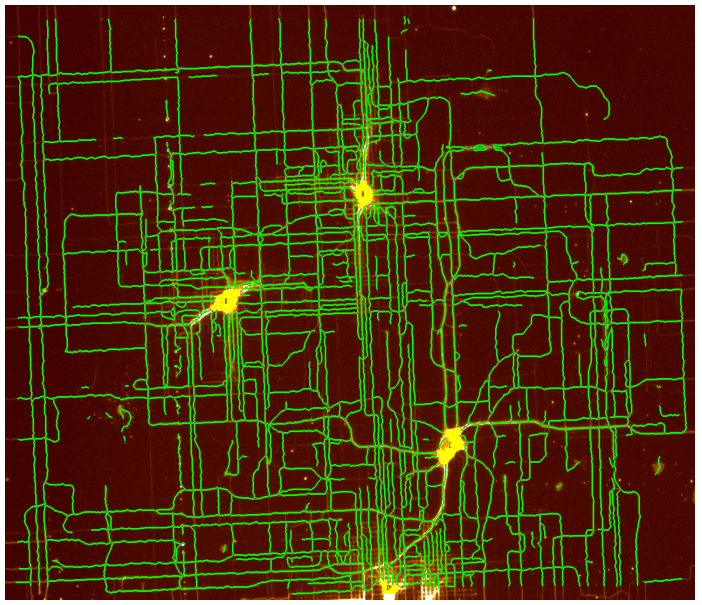

(A)

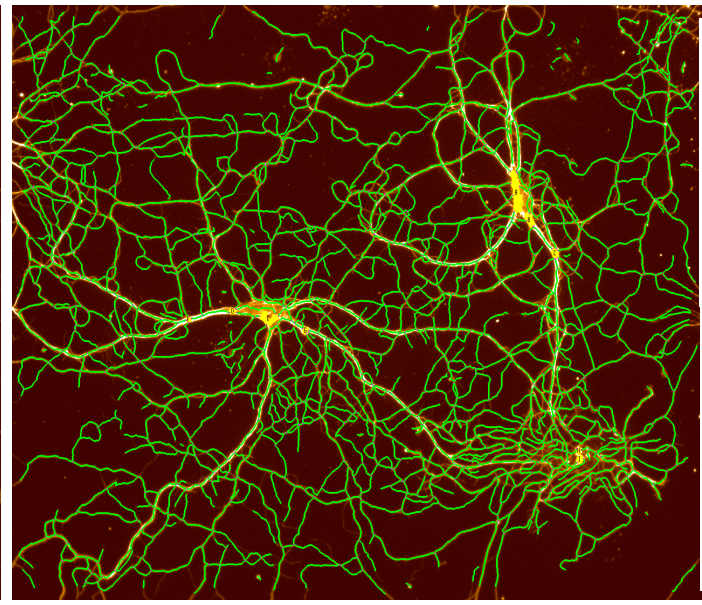

(B)

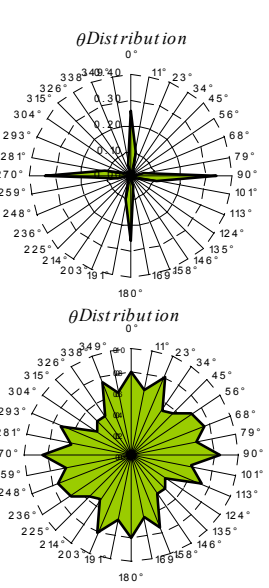

(C)

FIG 2: (A) Traces of neurons cultured on semiconductor surfaces with arrays of pillars. This image shows the distribution of $\beta$-III Tubulin in neurites. Note the growth directions. (B) Neurons cultured on a smooth semiconductor surface. Note the difference between growth directions and lengths with neurons shown in (A). (C) Quantitation of the neurite growth angles, obtained from automated traces of neurites grown on different topologies shown in (A) and (B), with the growth angles shown in the top and bottom radial plots respectively. 